\title{
Potential in spin-density-functional theory of noncollinear magnetism determined by the many-electron ground state
}

\begin{abstract}
Nikitas I. Gidopoulos*
ISIS Facility, Rutherford Appleton Laboratory, Chilton, Didcot, Oxon OX11 0QX, England, United Kingdom

(Received 29 September 2006; revised manuscript received 8 February 2007; published 11 April 2007)

Since spin-density-functional theory was first proposed, but also recently, examples were constructed to show that a spin potential may share its ground state with other spin potentials. In fact, for collinear magnetic fields and systems with fixed magnetization, the mapping between potentials and ground states is invertible, provided the magnetization is not saturated and that spin potentials are determined within a spin constant. We complete the proof that the mapping is invertible also for noncollinear magnetic fields and systems with more than one electron. We then discuss the noncollinear exchange and correlation energy functional in the localspin-density approximation and suggest improvements.
\end{abstract}

DOI: 10.1103/PhysRevB.75.134408

PACS number(s): 71.15.Mb, 31.15.Ew

\section{INTRODUCTION}

The remarkable success of density-functional theory (DFT),${ }^{1-4}$ as a tool for electronic structure calculations in solid-state physics and quantum chemistry, is owed not only to its efficiency but also to the soundness of the underlying principles. In fact, the quest to refine the foundations of the theory often results naturally in better understanding and improving the accuracy of the approximation. The aim of this work is to present such an example.

So far, it appeared that the sound basis of ground-state (g.s.) DFT is not shared by g.s. spin density-functional theory (SDFT), ${ }^{2,5-9}$ commonly used when the electronic system of interest lies in a weak external magnetic field, or even when there is no external magnetic field but the electrons in the system do not form closed shells. Although formally SDFT is a straightforward extension of the original theory, the analog of DFT's Hohenberg-Kohn (HK) theorem, ${ }^{1}$ establishing the 1-1 correspondence between the set of spin potentials $\{\mathcal{V}\}$ and the set of g.s. spin-densities $\{\varrho\}$, has not been established yet for noncollinear magnetic fields. By the spin potential we mean the potential and magnetic field $\mathcal{V}=(V ; \mathbf{B})$ or $\mathcal{V}=\left(V_{\uparrow \uparrow}, V_{\uparrow \downarrow}, V_{\downarrow \uparrow}, V_{\downarrow \downarrow}\right)$. The spin density represents the charge and magnetization densities $\varrho=(\rho ; \mathbf{m})$, or $\varrho=\left(\rho_{\uparrow \uparrow}, \rho_{\uparrow \downarrow}, \rho_{\downarrow \uparrow}, \rho_{\downarrow \downarrow}\right)$.

Traditionally, invertibility of the mapping between $\{\mathcal{V}\}$ and $\{\varrho\}$ is proven in two steps. First, showing that different spin potentials have different ground states, i.e., the mapping between the set of spin potentials $\{\mathcal{V}\}$ and the set of ground states $\{\Psi\}$ is invertible. Second, showing that different ground states, arising from different spin potentials, have different densities, i.e., that the mapping between $\{\Psi\}$ and $\{\varrho\}$ is also $1-1$.

It is straightforward to show that the second mapping is invertible. In the publication where von Barth and Hedin 5 proposed SDFT, they also constructed, for the single-electron g.s. $\Psi$ of any spin potential, a whole class of different (noncollinear) spin potentials that admitted $\Psi$ as an eigenstate, and under rather mild conditions as their g.s. (see, however, Ref. 10), concluding that the first step in the proof of the HK theorem could not hold in general. Recently, interest is revived on the same issue. ${ }^{11-16}$ In the case of collinear mag- netic fields, Capelle and Vignale ${ }^{11}$ gave several examples where the spin potential was not determined uniquely by the spin density. Eschrig and Pickett ${ }^{12}$ studied collinear and noncollinear magnetic fields. They distinguished two cases, i.e., when the g.s. is a pure-spin state or an impure-spin state. Pure-spin states are states which through a local rotation of the spin coordinates may be transformed to have a definite number of spin-up and -down electrons. Impure-spin states are states which cannot be transformed to have a definite number of spin-up and -down electrons under any local spin rotation. It was found that when the g.s. of the Hamiltonian $\hat{\mathcal{H}}$ is an impure-spin state, there is no ambiguity (apart from an overall constant) in determining the spin potential in $\hat{\mathcal{H}}$ by the ground state. However, they also discovered that purespin states are eigenstates of an operator which, in the laboratory unrotated spin space, corresponds to a generally noncollinear magnetic field with constant magnitude and concluded that the spin potential in $\hat{\mathcal{H}}$ cannot be determined uniquely by the ground state.

If these findings did hold, in general, the meaningfulness of the Kohn-Sham (KS) scheme would be questioned, since in the latter, the noninteracting $\mathrm{KS}$ spin potential is not known and must be determined together with the KS state that yields the g.s. spin density.

Recent work gives hope that the mapping is invertible. Numerical investigations by Ullrich ${ }^{16}$ on lattices give evidence that in the continuum limit and for noncollinear magnetic fields, nonuniqueness is very rare if not completely absent. Further, the study of finite temperature SDFT by Argaman and Makov, ${ }^{13}$ in the limit of vanishing temperature (provided the limit is not singular), shows that the mapping between $\mathcal{V}$ and $\varrho$ is invertible.

In the main part of the paper, we resolve the issue proving that the mapping is indeed invertible.

\section{Notation}

To proceed, consider the following generic spinHamiltonian $\hat{\mathcal{H}}$ employed in SDFT: 


$$
\hat{\mathcal{H}}=\hat{T}+\gamma \hat{V}^{\mathrm{ee}}+\hat{\mathcal{V}} .
$$

$\hat{T}$ and $\hat{V}^{\text {ee }}$ in Eq. (1) are the kinetic-energy and electron repulsion operators, $\gamma=1(0)$ for an interacting (noninteracting) system, and $\hat{\mathcal{V}}$ is the spin-potential operator.

The Hamiltonian $\hat{\mathcal{H}}$ is not gauge invariant. To restore gauge invariance, one should include the interaction of the current with the vector potential, which is ignored here. As it stands, $\hat{\mathcal{H}}$ is appropriate for weak magnetic fields.

In the following, we shall focus on the operators for the kinetic energy,

$$
\hat{T}=-\frac{\hbar^{2}}{2 m} \sum_{\tau=\uparrow, \downarrow} \int d \mathbf{r} \hat{\psi}_{\tau}^{\dagger}(\mathbf{r}) \nabla^{2} \hat{\psi}_{\tau}(\mathbf{r}),
$$

and the spin potential,

$$
\hat{\mathcal{V}}=\int d \mathbf{r}\left(\hat{\psi}_{\uparrow}^{\dagger}(\mathbf{r}) \hat{\psi}_{\downarrow}^{\dagger}(\mathbf{r})\right) \mathrm{V}_{\text {spin }}(\mathbf{r})\left(\begin{array}{c}
\hat{\psi}_{\uparrow}(\mathbf{r}) \\
\hat{\psi}_{\downarrow}(\mathbf{r})
\end{array}\right),
$$

where $\hat{\psi}_{\tau}^{\dagger}(\mathbf{r})$ and $\hat{\psi}_{\tau}(\mathbf{r})$ are second quantization creation and annihilation fermion field operators and $\mathrm{V}_{\text {spin }}(\mathbf{r})$ is the $2 \times 2$ matrix of the spin potential,

$$
\mathrm{V}_{\text {spin }}(\mathbf{r})=\left(\begin{array}{cc}
V_{\uparrow \uparrow}(\mathbf{r}) & V_{\uparrow \downarrow}(\mathbf{r}) \\
V_{\downarrow \uparrow}(\mathbf{r}) & V_{\downarrow \downarrow}(\mathbf{r})
\end{array}\right) .
$$

We may express the potential and magnetic field in terms of the spin-potential matrix elements as follows:

$$
\begin{gathered}
V(\mathbf{r})=\frac{V_{\uparrow \uparrow}(\mathbf{r})+V_{\downarrow \downarrow}(\mathbf{r})}{2}, \\
\mu_{0} B_{x}(\mathbf{r})=\frac{V_{\uparrow \downarrow}(\mathbf{r})+V_{\downarrow \uparrow}(\mathbf{r})}{2}, \\
\mu_{0} B_{y}(\mathbf{r})=\frac{V_{\uparrow \downarrow}(\mathbf{r})-V_{\downarrow \uparrow}(\mathbf{r})}{-2 i}, \\
\mu_{0} B_{z}(\mathbf{r})=\frac{V_{\uparrow \uparrow}(\mathbf{r})-V_{\downarrow \downarrow}(\mathbf{r})}{2} .
\end{gathered}
$$

Then, the matrix of the spin potential can be rewritten as

$$
\mathrm{V}_{\text {spin }}(\mathbf{r})=\left(\begin{array}{cc}
V(\mathbf{r})+\mu_{0} B_{z}(\mathbf{r}) & \mu_{0}\left(B_{x}(\mathbf{r})-i B_{y}(\mathbf{r})\right) \\
\mu_{0}\left(B_{x}(\mathbf{r})+i B_{y}(\mathbf{r})\right) & V(\mathbf{r})-\mu_{0} B_{z}(\mathbf{r})
\end{array}\right)
$$

or, in more compact form, ${ }^{17}$

$$
\mathrm{V}_{\text {spin }}(\mathbf{r})=V(\mathbf{r}) I+\mu_{0} \mathbf{B}(\mathbf{r}) \cdot \boldsymbol{\sigma} .
$$

I is the $2 \times 2$ unity matrix, and $\boldsymbol{\sigma}$ is the vector of the $2 \times 2$ Pauli spin matrices, $\sigma=\left(\sigma_{x}, \sigma_{y}, \sigma_{z}\right)$.

A consequence of Eq. (10) is that the operator for the spin potential can be split as the sum of the operators for the potential and the magnetic field as follows:

$$
\hat{\mathcal{V}}=\hat{V}+\hat{B},
$$

where the potential operator is given by

$$
\hat{V}=\int d \mathbf{r} \hat{\rho}(\mathbf{r}) V(\mathbf{r}),
$$

and the magnetic field operator is

$$
\hat{B}=-\int d \mathbf{r} \hat{\mathbf{m}}(\mathbf{r}) \cdot \mathbf{B}(\mathbf{r}) .
$$

$\hat{\rho}(\mathbf{r})$ is the charge density operator,

$$
\hat{\rho}(\mathbf{r})=\sum_{\tau=\uparrow, \downarrow} \hat{\psi}_{\tau}^{\dagger}(\mathbf{r}) \hat{\psi}_{\tau}(\mathbf{r}),
$$

and $\hat{\mathbf{m}}(\mathbf{r})$ is the magnetization density operator,

$$
\hat{\mathbf{m}}(\mathbf{r})=-\mu_{0}\left(\hat{\psi}_{\uparrow}^{\dagger}(\mathbf{r}) \hat{\psi}_{\downarrow}^{\dagger}(\mathbf{r})\right) \boldsymbol{\sigma}\left(\begin{array}{c}
\hat{\psi}_{\uparrow}(\mathbf{r}) \\
\hat{\psi}_{\downarrow}(\mathbf{r})
\end{array}\right) .
$$

To conclude the introduction on notation, the spin density $\varrho$ is given by a $2 \times 2$ matrix as follows:

$$
\varrho(\mathbf{r})=\left(\begin{array}{cc}
\rho_{\uparrow \uparrow}(\mathbf{r}) & \rho_{\uparrow \downarrow}(\mathbf{r}) \\
\rho_{\downarrow \uparrow}(\mathbf{r}) & \rho_{\downarrow \downarrow}(\mathbf{r})
\end{array}\right) .
$$

Here, too, we may write the charge and magnetization densities in terms of the spin-density matrix elements as follows:

$$
\begin{gathered}
\rho(\mathbf{r})=\rho_{\uparrow \uparrow}(\mathbf{r})+\rho_{\downarrow \downarrow}(\mathbf{r}), \\
m_{x}(\mathbf{r})=-\mu_{0}\left[\rho_{\uparrow \downarrow}(\mathbf{r})+\rho_{\downarrow \uparrow}(\mathbf{r})\right], \\
-i m_{y}(\mathbf{r})=-\mu_{0}\left[\rho_{\uparrow \downarrow}(\mathbf{r})-\rho_{\downarrow \uparrow}(\mathbf{r})\right], \\
m_{z}(\mathbf{r})=-\mu_{0}\left[\rho_{\uparrow \uparrow}(\mathbf{r})-\rho_{\downarrow \downarrow}(\mathbf{r})\right] .
\end{gathered}
$$

The matrix of the spin density can be written as ${ }^{17}$

$$
\varrho(\mathbf{r})=\frac{1}{2}\left[\rho(\mathbf{r}) \mid-\frac{1}{\mu_{0}} \mathbf{m}(\mathbf{r}) \cdot \boldsymbol{\sigma}\right] .
$$

\section{COLLINEAR MAGNETIC FIELDS}

We start by reviewing the collinear case. It is customary to define the direction of the $z$ axis along the magnetic field. We have $\mathbf{B}(\mathbf{r})=B_{z}(\mathbf{r}) \hat{\mathbf{z}}$, and the operator for the magnetic field is $\hat{B}=-\int d \mathbf{r} \hat{m}_{z}(\mathbf{r}) B_{z}(\mathbf{r})$, where $\hat{m}_{z}(\mathbf{r})=-\mu_{0}\left[\hat{\psi}_{\uparrow}^{\dagger}(\mathbf{r}) \hat{\psi}_{\uparrow}(\mathbf{r})\right.$ $\left.-\hat{\psi}_{\downarrow}^{\dagger}(\mathbf{r}) \hat{\psi}_{\downarrow}(\mathbf{r})\right]$.

There is no ambiguity regarding the invertibility of the mapping between potentials and ground states in SDFT for collinear fields. For the latter, the eigenstates of the Hamiltonian $\hat{\mathcal{H}}$ are pure-spin states, since the spin-up and spindown particle-number operators $\hat{N}_{\uparrow}$ and $\hat{N}_{\downarrow}$,

$$
\hat{N}_{\tau} \doteq \int d \mathbf{r} \hat{\psi}_{\tau}^{\dagger}(\mathbf{r}) \hat{\psi}_{\tau}(\mathbf{r}), \quad \tau=\uparrow, \downarrow,
$$

independently commute with $\hat{\mathcal{H}}$.

Capelle and Vignale ${ }^{11}$ discovered two cases of freedom in determining the spin potential. First, as in DFT where the trivial density $\rho(\mathbf{r})=0$ cannot determine the potential $V(\mathbf{r})$, in 
the same way here a trivial component of the spin density cannot determine the corresponding component of the spin potential, i.e., for a system of electrons that are all spin polarized in one direction, say, $\rho_{\uparrow}(\mathbf{r})>0, \rho_{\downarrow}(\mathbf{r})=0$, the spin component of the potential in the other direction, $V_{\downarrow}(\mathbf{r})$, is completely arbitrary (as long as the system remains fully polarized). This kind of indeterminacy cannot have any practical consequence as there are no electrons to experience the arbitrariness.

Second, together with the freedom of a constant shift in the potential (because $\hat{N}_{\uparrow}+\hat{N}_{\downarrow}$ commutes with $\hat{\mathcal{H}}$ ), there is an additional freedom of a constant shift in the magnetic field, $B_{0}$ appearing because the magnetization operator $\hat{N}_{\uparrow}-\hat{N}_{\downarrow}$ commutes with $\hat{\mathcal{H}}$. According to Capelle and Vignale, this is an example of a systematic nonuniqueness arising from a conserved quantity (as opposed to an accidental nonuniqueness such as the example of the perfectly spin-polarized system.) The formulation of SDFT can be corrected to respect this freedom by constraining, at the functional differentiations, both the charge and the magnetization densities to integrate separately to a fixed number of particles. ${ }^{14}$ Different KS calculations must be performed for systems with fixed magnetizations $N^{\uparrow}-N^{\downarrow}$ (fixed spin moment calculations), and the g.s. is given by the system with the lowest KS total energy. ${ }^{14}$

Still, the challenging question in collinear SDFT, in order to establish the 1-1 mapping, is whether this is all the freedom available or whether there could be other kinds of nonuniqueness present. It was shown that for continuous spin potentials, this does not happen. ${ }^{12,14}$ For completeness of presentation, the main points of the author's proof ${ }^{14}$ are included below.

\section{Proof that different spin potentials have different eigenstates}

For collinear magnetic fields, we consider two spin potentials as different if they differ by more than a spin constant.

Consider now a continuous diagonal spin potential (corresponding to a collinear magnetic field in the $z$ direction)

$$
\mathrm{V}_{\text {spin }}^{d}(\mathbf{r})=\left(\begin{array}{cc}
V_{\uparrow}(\mathbf{r}) & 0 \\
0 & V_{\downarrow}(\mathbf{r})
\end{array}\right) \text {. }
$$

We shall denote the diagonal spin potential by $\left(V_{\uparrow}, V_{\downarrow}\right)$. The spin-potential operator [Eq. (3)] becomes

$$
\hat{\mathcal{V}}^{d}=\hat{V}_{\uparrow}+\hat{V}_{\downarrow},
$$

with

$$
\hat{V}_{\tau}=\int d \mathbf{r} V_{\tau}(\mathbf{r}) \hat{\psi}_{\tau}^{\dagger}(\mathbf{r}) \hat{\psi}_{\tau}(\mathbf{r}), \quad \tau=\uparrow, \downarrow .
$$

$\Psi$ is an eigenstate of the spin-diagonal Hamiltonian

$$
\hat{\mathcal{H}}^{d}=\hat{T}+\gamma \hat{V}^{\mathrm{ee}}+\hat{V}_{\uparrow}+\hat{V}_{\downarrow} .
$$

$\gamma=1(0)$ for an interacting (noninteracting) system.

$\hat{\mathcal{H}}^{d}$ commutes with $\hat{N}^{\uparrow}$ and $\hat{N}^{\downarrow}$, and in the (assumed) absence of degeneracy, $\Psi$ describes a state with a definite num- ber of spin-up $N^{\uparrow}$ and spin-down $N^{\downarrow}$ electrons $\left(N^{\uparrow}+N^{\downarrow}=N\right)$. It also holds that $N^{\uparrow}, N^{\downarrow} \neq 0$, i.e., the system is not completely spin polarized.

THEOREM 1. There is no other continuous potential $\left(V_{\uparrow}^{\prime}, V_{\downarrow}^{\prime}\right)$ which has the same state $\Psi$ as eigenstate and whose components differ from $\left(V_{\uparrow}, V_{\downarrow}\right)$ by more than a spindependent constant.

Proof. Assume the contrary that a continuous diagonal spin potential $\left(V_{\uparrow}^{\prime}, V_{\downarrow}^{\prime}\right)$ exists having $\Psi$ as an eigenstate and differing from $\left(V_{\uparrow}, V_{\downarrow}\right)$ by more than a spin constant.

The two Schrödinger equations are

$$
\begin{aligned}
& {\left[\hat{T}+\gamma \hat{V}^{\mathrm{ee}}+\sum_{\tau=\uparrow, \downarrow} \hat{V}_{\tau}\right]|\Psi\rangle=E|\Psi\rangle,} \\
& {\left[\hat{T}+\gamma \hat{V}^{\mathrm{ee}}+\sum_{\tau=\uparrow, \downarrow} \hat{V}_{\tau}^{\prime}\right]|\Psi\rangle=E^{\prime}|\Psi\rangle .}
\end{aligned}
$$

Shift the potentials $\left(V_{\uparrow}, V_{\downarrow}\right)$ and $\left(V_{\uparrow}^{\prime}, V_{\downarrow}^{\prime}\right)$ by constants $-E / N$ and $-E^{\prime} / N$ and subtract the two equations to obtain

$$
\sum_{\tau=\uparrow, \downarrow} \delta \hat{V}_{\tau}|\Psi\rangle=0,
$$

where $\delta \hat{V}_{\tau}=\hat{V}_{\tau}^{\prime}-\hat{V}_{\tau}$. We assumed that $\delta V_{\tau}(\mathbf{r})$ is not a constant.

Take the inner product of the left-hand side of Eq. (29) with $\langle 0| \hat{\psi}_{\downarrow}\left(\mathbf{r}_{N}\right) \cdots \hat{\psi}_{\downarrow}\left(\mathbf{r}_{N_{\uparrow}+1}\right) \hat{\psi}_{\uparrow}\left(\mathbf{r}_{N_{\uparrow}}\right) \cdots \hat{\psi}_{\uparrow}\left(\mathbf{r}_{1}\right)$. Doing the commutators, we find

$$
\begin{aligned}
& {\left[\sum_{i=1}^{N^{\uparrow}} \delta V^{\uparrow}\left(\mathbf{r}_{i}\right)+\sum_{j=N^{\uparrow}+1}^{N} \delta V^{\downarrow}\left(\mathbf{r}_{j}\right)\right] \Psi\left(\mathbf{r}_{1}, \ldots, \mathbf{r}_{N^{\dagger}} ; \mathbf{r}_{N^{\uparrow}+1}, \ldots, \mathbf{r}_{N}\right)} \\
& \quad=0
\end{aligned}
$$

where

$$
\begin{aligned}
& \Psi\left(\mathbf{r}_{1}, \ldots, \mathbf{r}_{N_{\uparrow}} ; \mathbf{r}_{N_{\uparrow}+1}, \ldots, \mathbf{r}_{N}\right) \\
& =\frac{\left\langle 0\left|\hat{\psi}_{\downarrow}\left(\mathbf{r}_{N}\right) \cdots \hat{\psi}_{\downarrow}\left(\mathbf{r}_{N_{\uparrow}+1}\right) \hat{\psi}_{\uparrow}\left(\mathbf{r}_{N_{\uparrow}}\right) \cdots \hat{\psi}_{\uparrow}\left(\mathbf{r}_{1}\right)\right| \Psi\right\rangle}{\sqrt{N_{\uparrow} ! N_{\downarrow} !}} .
\end{aligned}
$$

In the following, we denote by $\Psi\left(\mathbf{r}_{1}, \ldots, \mathbf{r}_{N}\right)$ the wave function in Eq. (31). The normalized wave function $\Psi\left(\mathbf{r}_{1}, \ldots, \mathbf{r}_{N}\right)$ is antisymmetric in $\mathbf{r}_{i}, 1 \leqslant i \leqslant N_{\uparrow}$, and $\mathbf{r}_{j}, N_{\uparrow}+1 \leqslant j \leqslant N$, but not with respect to an interchange of a "spin-up" and a "spindown" coordinate $\mathbf{r}_{i}$ and $\mathbf{r}_{j} . \Psi\left(\mathbf{r}_{1}, \ldots, \mathbf{r}_{N}\right)$ has the same spin-up and spin-down densities as $\Psi$ and satisfies the following Schrödinger equation:

$$
\begin{aligned}
& {\left[-\frac{\hbar^{2}}{2 m} \sum_{k=1}^{N} \nabla_{j}^{2}+\sum_{i=1}^{N^{\uparrow}} V^{\uparrow}\left(\mathbf{r}_{i}\right)+\sum_{j=N^{\uparrow}+1}^{N} V^{\downarrow}\left(\mathbf{r}_{j}\right)\right.} \\
& \left.\quad+\frac{\gamma}{2} \sum_{k \neq l=1}^{N} \frac{1}{r_{k l}}\right] \Psi\left(\mathbf{r}_{1}, \ldots, \mathbf{r}_{N}\right)=E \Psi\left(\mathbf{r}_{1}, \ldots, \mathbf{r}_{N}\right) .
\end{aligned}
$$

Hence, $\Psi\left(\mathbf{r}_{1}, \ldots, \mathbf{r}_{N}\right)$ can be interpreted to describe a twocomponent system of spin-1/2 fermions. 
Consider a region $\mathcal{R}_{N}$ in $\mathrm{R}^{3 N}$, where $\mathrm{R}$ is the real axis, with the property that $\Psi\left(\mathbf{r}_{1}, \ldots, \mathbf{r}_{N}\right)$ is nonzero for all $\left\{\mathbf{r}_{1}, \ldots, \mathbf{r}_{N^{\uparrow}} ; \mathbf{r}_{N^{\uparrow}+1}, \ldots, \mathbf{r}_{N}\right\}$ belonging in $\mathcal{R}_{N}$. Divide Eq. (30) through by $\Psi\left(\mathbf{r}_{1}, \ldots, \mathbf{r}_{N}\right)$ to get

$$
\sum_{i=1}^{N_{\uparrow}} \delta V_{\uparrow}\left(\mathbf{r}_{i}\right)+\sum_{j=N_{\uparrow}+1}^{N} \delta V_{\downarrow}\left(\mathbf{r}_{j}\right)=0
$$

for all $\mathbf{r}_{i}, \mathbf{r}_{j}$ which form points $\left\{\mathbf{r}_{1}, \ldots, \mathbf{r}_{N_{\uparrow}} ; \mathbf{r}_{N_{\uparrow}+1}, \ldots, \mathbf{r}_{N}\right\}$ of $\mathcal{R}_{N}$. Since the two terms on the left-hand side in Eq. (33) correspond to different coordinates, we must have that each term separately equals a constant: $\sum_{i=1}^{N_{\uparrow}} \delta V_{\uparrow}\left(\mathbf{r}_{i}\right)=C$ and $\sum_{j=N_{\uparrow}+1}^{N} \delta V_{\downarrow}\left(\mathbf{r}_{j}\right)=-C$. By the same argument, $\delta V_{\uparrow}\left(\mathbf{r}_{i}\right)=C / N_{\uparrow}$ and $\delta V_{\downarrow}\left(\mathbf{r}_{j}\right)=-C / N_{\downarrow}$ for any admissible $\mathbf{r}_{i}, \mathbf{r}_{j}$.

The region where $\Psi$ is nonzero is bounded by a nodal surface, so $\mathrm{R}^{3 N}$ is separated in disconnected regions $\mathcal{R}_{N}$ and the corresponding constants $C$ may be different in the different regions.

The vectors $\mathbf{r}_{i}$ and $\mathbf{r}_{j}$ which, compose the $3 N$-dimensional vectors of $\mathcal{R}_{N}$, will form the regions $\mathcal{R}^{\uparrow}$ and $\mathcal{R}^{\downarrow}$ in $\mathrm{R}^{3}$ : $\mathbf{r}_{i} \in \mathcal{R}^{\uparrow}$ and $\quad \mathbf{r}_{j} \in \mathcal{R}^{\downarrow}$. Vectors $\quad \mathbf{r}_{i}$ and $\mathbf{r}_{j}$ composing $3 N$-dimensional vectors in a different region $\mathcal{R}_{N}^{\prime}$ will form different, in general, regions $\mathcal{R}^{\uparrow \prime}$ and $\mathcal{R}^{\downarrow \prime}$ of $\mathrm{R}^{3}$. If either $\mathcal{R}^{\uparrow}$ and $\mathcal{R}^{\uparrow \prime}$ or $\mathcal{R}^{\downarrow}$ and $\mathcal{R}^{\downarrow \prime}$ have common points, then the constants $C$ and $C^{\prime}$ will be the same. There must be two $\mathcal{R}^{\uparrow}$ and $\mathcal{R}^{\uparrow \prime}$ with no common points, or else $\delta V_{\uparrow}(\mathbf{r}), \delta V_{\downarrow}(\mathbf{r})$ would equal two constants, which is absurd. Then, there must be two such regions which are neighboring, because all $\mathrm{R}^{3}$ must be covered. Hence, for points $\mathbf{r}, \mathbf{r}^{\prime}$ belonging in different $\mathcal{R}^{\uparrow}$ and $\mathcal{R}^{\uparrow \prime}$ and which are arbitrarily close, $\delta V_{\uparrow}(\mathbf{r})=C / N_{\uparrow}$ and $\delta V_{\uparrow}\left(\mathbf{r}^{\prime}\right)=C^{\prime} / N_{\uparrow}$, which implies that the constants $C$ and $C^{\prime}$ must be equal, which is absurd. The constants must be equal because, otherwise, the potential $V_{\uparrow}^{\prime}$ would be discontinuous, as it would be equal to $V_{\uparrow}(\mathbf{r})+C / N_{\uparrow}$ and to $V_{\uparrow}\left(\mathbf{r}^{\prime}\right)+C^{\prime} / N_{\uparrow}$ for points $\mathbf{r}, \mathbf{r}^{\prime}$, which lie arbitrarily close. So, the proof is complete.

The similarity between the formulations of SDFT and of two-component DFT (Ref. 18) is noteworthy.

\section{NONCOLLINEAR MAGNETIC FIELDS}

\section{A. The example by von Barth and Hedin}

The general case of noncollinear SDFT is not very different. We focus first on the example of von Barth and Hedin. For any single-electron state $\Psi$, they constructed a singleparticle spin potential $\mathcal{V}^{\prime}$, which acting on $\Psi$ gives zero,

$$
\mathcal{V}^{\prime} \Psi=0
$$

Hence, if $\Psi$ is the ground state of a spin Hamiltonian $\hat{\mathcal{H}}$, then $\Psi$ will be an eigenstate of $\hat{\mathcal{H}}+\lambda \mathcal{V}^{\prime}$. A whole class of $\mathcal{V}^{\prime}$ was found, and it was argued that small enough $\lambda$ must exist for which $\Psi$ is the g.s. of $\hat{\mathcal{H}}+\lambda \mathcal{V}^{\prime}$. von Barth and Hedin did not explain how they constructed $\mathcal{V}^{\prime}$, but it is easy to do so: any single-particle state $\Psi$ with spinor wave function

$$
\left(\begin{array}{l}
\psi_{\uparrow}(\mathbf{r}) \\
\psi_{\downarrow}(\mathbf{r})
\end{array}\right)
$$

defines its own (local) orientation in spin space, call it spin up,

$$
|\uparrow\rangle=\left[\left|\psi_{\uparrow}(\mathbf{r})\right|^{2}+\left|\psi_{\downarrow}(\mathbf{r})\right|^{2}\right]^{-1 / 2}\left(\begin{array}{l}
\psi_{\uparrow}(\mathbf{r}) \\
\psi_{\downarrow}(\mathbf{r})
\end{array}\right) .
$$

The spin-down orientation is also defined as follows:

$$
|\downarrow\rangle=\left[\left|\psi_{\uparrow}(\mathbf{r})\right|^{2}+\left|\psi_{\downarrow}(\mathbf{r})\right|^{2}\right]^{-1 / 2}\left(\begin{array}{c}
\psi_{\downarrow}^{*}(\mathbf{r}) \\
-\psi_{\uparrow}^{*}(\mathbf{r})
\end{array}\right) .
$$

One can construct single-particle, spin-up, $|\uparrow\rangle\langle\uparrow|$, and spindown, $|\downarrow\rangle\langle\downarrow|$, number operators which have $\Psi$ as their eigenstate,

$$
|\uparrow\rangle\langle\uparrow \mid \Psi\rangle=|\Psi\rangle, \quad|\downarrow\rangle\langle\downarrow \mid \Psi\rangle=0 .
$$

So, $\Psi$ is an eigenstate of the single-particle spin-down number operator $|\downarrow\rangle\langle\downarrow|$ with eigenvalue zero, and for any $V^{\prime}(\mathbf{r})$, the spin potential $\mathcal{V}^{\prime}=V^{\prime}(\mathbf{r})|\downarrow\rangle\langle\downarrow|$ maps $\Psi$ to zero. It is evident that this example is a generalization to the noncollinear case of the accidental nonuniqueness for collinear systems fully saturated in one spin direction, leaving the potential in the other spin direction undetermined. ${ }^{13}$ In order to extend the example of von Barth and Hedin to many-electron systems, one would need to construct fully spin-polarized purespin states and therefore to obtain a local rotation in spin space that reduces $\hat{\mathcal{H}}$ to spin-diagonal form.

\section{B. Can the Hamiltonian $\hat{\mathcal{H}}$ be spin diagonalized?}

We point out that the discovery by Eschrig and Pickett relies exactly on the assumption that this is possible. In fact, criticism on the reasoning by Eschrig and Pickett (footnote 18 in Ref. 13), misses the point, as it is restricted to Hamiltonians that cannot be transformed to spin-diagonal form.

So, given the proof ${ }^{12}$ that impure-spin states do not lead to any kind of nonuniqueness, the question about the invertibility of the mapping between spin potentials and ground states for noncollinear spin Hamiltonians boils down to the existence or not of a local transformation that spin diagonalizes $\hat{\mathcal{H}}$. We consequently prove the following:

THEOREM 2. The Hamiltonian $\hat{\mathcal{H}}$ in Eq. (1) cannot be transformed to spin-diagonal form, unless the magnetic field is collinear.

Proof. We introduce the general unitary transformation that rotates locally, at every point in real space, the spin degrees of freedom,

$$
\mathrm{U}(\mathbf{r})=\left(\begin{array}{cc}
e^{i \theta(\mathbf{r})} \cos \omega(\mathbf{r}) & -e^{i \phi(\mathbf{r})} \sin \omega(\mathbf{r}) \\
e^{-i \phi(\mathbf{r})} \sin \omega(\mathbf{r}) & e^{-i \theta(\mathbf{r})} \cos \omega(\mathbf{r})
\end{array}\right) .
$$

$\theta(\mathbf{r}), \phi(\mathbf{r})$, and $\omega(\mathbf{r})$ are real functions. We have

$$
\mathrm{U}(\mathbf{r}) \mathrm{U}^{\dagger}(\mathbf{r})=\mathrm{U}^{\dagger}(\mathbf{r}) \mathrm{U}(\mathbf{r})=\mathrm{I} .
$$

Rotated in spin space, the second quantized fermion field operators can be defined as follows: 


$$
\begin{aligned}
\left(\hat{\Psi}_{\uparrow}^{\dagger}(\mathbf{r}) \hat{\Psi}_{\downarrow}^{\dagger}(\mathbf{r})\right) & =\left(\hat{\psi}_{\uparrow}^{\dagger}(\mathbf{r}) \hat{\psi}_{\downarrow}^{\dagger}(\mathbf{r})\right) U^{\dagger}(\mathbf{r}), \\
\left(\begin{array}{c}
\hat{\Psi}_{\uparrow}(\mathbf{r}) \\
\hat{\Psi}_{\downarrow}(\mathbf{r})
\end{array}\right) & =U(\mathbf{r})\left(\begin{array}{c}
\hat{\psi}_{\uparrow}(\mathbf{r}) \\
\hat{\psi}_{\downarrow}(\mathbf{r})
\end{array}\right) .
\end{aligned}
$$

To familiarize ourselves with the notation, it is straightforward to confirm that the charge density operator $\hat{\rho}(\mathbf{r})$ is rotation (gauge) invariant:

$$
\begin{aligned}
\hat{\rho}(\mathbf{r}) & =\left(\hat{\psi}_{\uparrow}^{\dagger}(\mathbf{r}) \hat{\psi}_{\downarrow}^{\dagger}(\mathbf{r})\right)\left(\begin{array}{c}
\hat{\psi}_{\uparrow}(\mathbf{r}) \\
\hat{\psi}_{\downarrow}(\mathbf{r})
\end{array}\right) \\
& =\left(\hat{\psi}_{\uparrow}^{\dagger}(\mathbf{r}) \hat{\psi}_{\downarrow}^{\dagger}(\mathbf{r})\right) U^{\dagger}(\mathbf{r}) \mathrm{U}(\mathbf{r})\left(\begin{array}{c}
\hat{\psi}_{\uparrow}(\mathbf{r}) \\
\hat{\psi}_{\downarrow}(\mathbf{r})
\end{array}\right) \\
& =\left(\hat{\Psi}_{\uparrow}^{\dagger}(\mathbf{r}) \hat{\Psi}_{\downarrow}^{\dagger}(\mathbf{r})\right)\left(\begin{array}{c}
\hat{\Psi}_{\uparrow}(\mathbf{r}) \\
\hat{\Psi}_{\downarrow}(\mathbf{r})
\end{array}\right) .
\end{aligned}
$$

Is there a $U$ that diagonalizes $\hat{\mathcal{H}}$ in spin space?

A rotation in spin space may transform locally the magnetic field operator $\hat{b}_{\cup}$ to diagonal form. $\hat{V}$ and $\hat{V}^{\text {ee }}$ are independent of spin and do not change form. However, the kinetic-energy operator $\hat{T}$ in Eq. (2) is not invariant under the gauge transformation [Eq. (39)] and the transformed operator represents the motion of a particle in a gauge vector potential $\mathbf{A}(\mathbf{r})$ in spin space:

$$
\hat{T}=\frac{\hbar^{2}}{2 m} \int d \mathbf{r}\left(\hat{\Psi}_{\uparrow}^{\dagger}(\mathbf{r}) \hat{\Psi}_{\downarrow}^{\dagger}(\mathbf{r})\right)[-i \boldsymbol{\nabla}+\mathbf{A}]^{2}\left(\begin{array}{c}
\hat{\Psi}_{\uparrow}(\mathbf{r}) \\
\hat{\Psi}_{\downarrow}(\mathbf{r})
\end{array}\right),
$$

where $\mathbf{A}(\mathbf{r})=-i \mathrm{U}(\mathbf{r}) \boldsymbol{\nabla} \mathbf{U}^{\dagger}(\mathbf{r})$.

We further consider the rotated spin-up and down number operators,

$$
\hat{N}_{\mathrm{U}_{\tau}} \doteq \int d \mathbf{r} \hat{\Psi}_{\tau}^{\dagger}(\mathbf{r}) \hat{\Psi}_{\tau}(\mathbf{r}), \quad \tau=\uparrow, \downarrow,
$$

and the magnetization operator in rotated spin space,

$$
\hat{b}_{\mathrm{U}}=\hat{N}_{\mathrm{U} \uparrow}-\hat{N}_{\mathrm{U} \downarrow} \text {. }
$$

Rotating back to the original spin space, we see that $\hat{b}_{\cup}$ is the operator of a magnetic field $\mathbf{b}_{\cup}(\mathbf{r}) / \mu_{0}$ [Eq. (13)]:

$$
\hat{b}_{U}=-\int d \mathbf{r} \hat{\mathbf{m}}(\mathbf{r}) \cdot \frac{\mathbf{b}_{\mathrm{U}}(\mathbf{r})}{\mu_{0}} .
$$

$\mathbf{b}_{\cup}(\mathbf{r})$ is, in general, noncollinear,

$$
\begin{gathered}
b_{\cup x}(\mathbf{r})=-\sin 2 \omega(\mathbf{r}) \cos (\theta(\mathbf{r})-\phi(\mathbf{r})), \\
b_{\cup y}(\mathbf{r})=-\sin 2 \omega(\mathbf{r}) \sin (\theta(\mathbf{r})-\phi(\mathbf{r})), \\
b_{\cup z}(\mathbf{r})=+\cos 2 \omega(\mathbf{r}),
\end{gathered}
$$

and has unit magnitude $b_{\cup}^{2}(\mathbf{r})=1$.

We obtain tautologically the argument of Eschrig and Pickett. A pure-spin state (in rotated spin space) is by definition an eigenstate of the rotated magnetization $\hat{b}_{\mathrm{U}}$, which in the unrotated spin space represents a noncollinear magnetic field with constant magnitude. Of course, the question is whether pure-spin states exist, or equivalently whether an operator $\hat{b}_{\cup}$ exists that commutes with $\hat{\mathcal{H}} .{ }^{19}$ In that case, $\hat{\mathcal{H}}$ could be brought to spin-diagonal form. Indeed, in Ref. 12, it is explicitly assumed that such $\hat{b}_{\cup}$ exists $\left[\hat{b}_{\cup}\right.$ is denoted $\hat{U}_{o}$, defined by Eq. (15) in (Ref. 12)].

To answer the question, we need the commutator $\left[\hat{\mathcal{H}}, \hat{b}_{\cup}\right]$. We have $\left[\hat{V}^{\mathrm{ee}}, \hat{b}_{\mathrm{U}}\right]=\left[\hat{V}, \hat{b}_{\mathrm{U}}\right]=0$ and

$$
\begin{aligned}
& {\left[\hat{T}, \hat{b}_{\cup}\right]=-i \hbar \sum_{\alpha=x, y, z} \int d \mathbf{r} \hat{\mathbf{j}}_{\alpha}(\mathbf{r}) \cdot \nabla b_{\cup \alpha}(\mathbf{r}),} \\
& {\left[\hat{B}, \hat{b}_{\cup}\right]=-4 i \int d \mathbf{r} \hat{\mathbf{m}}(\mathbf{r}) \cdot \frac{\mathbf{B}(\mathbf{r}) \times \mathbf{b}_{\cup}(\mathbf{r})}{\mu_{0}},}
\end{aligned}
$$

where

$$
\begin{aligned}
\hat{\mathbf{j}}_{\alpha}(\mathbf{r})= & \frac{i \hbar}{2 m}\left\{\left[\boldsymbol{\nabla}\left(\hat{\psi}_{\uparrow}^{\dagger}(\mathbf{r}) \hat{\psi}_{\downarrow}^{\dagger}(\mathbf{r})\right)\right] \sigma_{\alpha}\left(\begin{array}{c}
\hat{\psi}_{\uparrow}(\mathbf{r}) \\
\hat{\psi}_{\downarrow}(\mathbf{r})
\end{array}\right)\right. \\
& \left.-\left(\hat{\psi}_{\uparrow}^{\dagger}(\mathbf{r}) \hat{\psi}_{\downarrow}^{\dagger}(\mathbf{r})\right) \sigma_{\alpha}\left[\nabla\left(\begin{array}{c}
\hat{\psi}_{\uparrow}(\mathbf{r}) \\
\hat{\psi}_{\downarrow}(\mathbf{r})
\end{array}\right)\right]\right\}, \quad \alpha=x, y, z .
\end{aligned}
$$

The two commutators correspond to operators for different physical quantities; $i\left[\hat{T}, \hat{b}_{\cup}\right]$ describes the interaction of currents with vector potentials and $i\left[\hat{B}, \hat{b}_{U}\right]$ the energy of a magnetic moment in a magnetic field, and hence they cannot cancel each other. In order that $\left[\hat{\mathcal{H}}, \hat{b}_{U}\right]=0$, each commutator must vanish separately. From Eq. (48), we have $\boldsymbol{\nabla} b_{\cup x, y, z}(\mathbf{r})$ $=0$, i.e., $\mathbf{b}_{\cup}$ is independent of $\mathbf{r}$. From (49), we have $\mathbf{B}(\mathbf{r})$ $\times \mathbf{b}_{\cup}=0$ and $\mathbf{B}(\mathbf{r})$ must be collinear, as it has to be parallel to $\mathbf{b}_{\mathrm{U}}$, which does not depend on $\mathbf{r}$.

Hence, in the noncollinear case, the Hamiltonian cannot be spin diagonalized by a rotation in spin space, and for many-electron systems, the mapping between spin potentials and ground states is invertible. ${ }^{19}$

\section{NONCOLLINEAR EXCHANGE AND CORRELATION ENERGY FUNCTIONAL}

An obvious challenge, since the spin-density matrix [Eq. (16)] for the uniform electron-gas model has vanishing offdiagonal matrix elements, is how to obtain the equivalent of the local-spin-density approximation (LSDA) and generalized-gradient approximation (GGA) for the exchange and correlation energy functional $E_{x c}$ in the noncollinear case. The problem was tackled elegantly by employing collinear functionals to simulate the noncollinear $E_{x c}{ }^{8,9,21}$

The magnetization density [Eq. (21)] is rotated to locally spin-diagonal form, ${ }^{17}$

$$
\mathbf{m}(\mathbf{r}) \cdot \boldsymbol{\sigma} \rightarrow \mathrm{U}(\mathbf{r}) \mathbf{m}(\mathbf{r}) \cdot \boldsymbol{\sigma} \bigcup^{\dagger}(\mathbf{r})=M_{z}(\mathbf{r}) \sigma_{z} .
$$

Then, the locally diagonal spin magnetization $M_{z}(\mathbf{r})$ is viewed momentarily as if it were the magnetization density 
of a collinear system. The corresponding (exchange and correlation) collinear magnetic field $B_{z}(\mathbf{r})$ is found by taking the functional derivative of the collinear exchange and correlation functional $E_{x c}^{0}$ :

$$
B_{z}(\mathbf{r})=\frac{\delta E_{x c}^{0}\left[\rho, M_{z}\right]}{\delta M_{z}(\mathbf{r})} .
$$

Then, $B_{z}(\mathbf{r})$ (seen as the magnetic field that would have resulted by properly taking the functional derivative $\delta E_{x c}\left[\rho, M_{z}\right] / \delta M_{z}(\mathbf{r})$ of the noncollinear functional) is rotated back, ${ }^{17}$

$$
B_{z}(\mathbf{r}) \sigma_{z} \rightarrow \mathrm{U}^{\dagger}(\mathbf{r}) B_{z}(\mathbf{r}) \sigma_{z} \mathrm{U}(\mathbf{r})=\mathbf{B}(\mathbf{r}) \cdot \boldsymbol{\sigma},
$$

to obtain the noncollinear magnetic field $\mathbf{B}(\mathbf{r})$ [Eq. (10)]. Finally, Pauli-like KS equations are solved using B(r). Perturbative corrections (spin stiffness) for the noncollinear exchange energy have also been proposed. ${ }^{21}$ The method has given access, successfully, to a wide range of systems exhibiting noncollinear magnetism, ${ }^{8,9}$ notably the spiral spindensity-wave g.s. of $\gamma$-Fe. ${ }^{22,23}$

However, formally, the noncollinear $E_{x c}\left[\rho, M_{z}\right]$ rather than the collinear exchange and correlation energy should have been used for the functional derivative [Eq. (52)]. The difference is the absence of the vector potential in $E_{x c}^{0}\left[\rho, M_{z}\right]$. We shall investigate the effect of this omission for weak $A$ and we shall derive a correction in first order [Eq. (69)] for $E_{x c}^{0}\left[\rho, M_{z}\right]$. The correction should describe the effect of $\mathrm{A}$ fairly accurately, since the quality of the results, so far, indicates that probably $\mathrm{A}$ is a weak perturbation.

Following Korenman et al. ${ }^{20}$ the transformed kineticenergy operator [Eq. (43)] can be expressed as a sum of three terms as follows:

$$
\hat{T}=\hat{T}_{0}+\hat{T}_{\mathrm{A}}+\hat{V}_{\mathrm{A}},
$$

where $\hat{T}_{0}$ has the familiar form [Eq. (2)] in terms of the rotated field operators [Eq. (41) and (42)],

$$
\hat{T}_{0}=-\frac{\hbar^{2}}{2 m} \sum_{\tau=\uparrow, \downarrow} \int d \mathbf{r} \hat{\Psi}_{\tau}^{\dagger}(\mathbf{r}) \nabla^{2} \hat{\Psi}_{\tau}(\mathbf{r}) .
$$

$\hat{T}_{\mathbf{A}}$ is linear in $\mathbf{A}$ and describes the interaction of $\mathbf{A}$ with spin currents,

$$
\hat{T}_{\mathrm{A}}=\hat{T}_{\mathrm{Ax}}+\hat{T}_{\mathrm{Ay}}+\hat{T}_{\mathrm{Az}},
$$

with

$$
\hat{T}_{\mathrm{A} \alpha}=\hbar \int d \mathbf{r} \hat{\mathbf{J}}_{\alpha}(\mathbf{r}) \cdot \mathbf{A}_{\alpha}(\mathbf{r}), \quad \alpha=x, y, z
$$

where the spin-current density operators are given by

$$
\begin{aligned}
\hat{\mathbf{J}}_{\alpha}(\mathbf{r})= & \frac{i \hbar}{2 m}\left\{\left[\nabla\left(\hat{\Psi}_{\uparrow}^{\dagger}(\mathbf{r}) \hat{\Psi}_{\downarrow}^{\dagger}(\mathbf{r})\right)\right] \sigma_{\alpha}\left(\begin{array}{c}
\hat{\Psi}_{\uparrow}(\mathbf{r}) \\
\hat{\Psi}_{\downarrow}(\mathbf{r})
\end{array}\right)\right. \\
& \left.-\left(\hat{\Psi}_{\uparrow}^{\dagger}(\mathbf{r}) \hat{\Psi}_{\downarrow}^{\dagger}(\mathbf{r})\right) \sigma_{\alpha}\left[\nabla\left(\begin{array}{c}
\hat{\Psi}_{\uparrow}(\mathbf{r}) \\
\hat{\Psi}_{\downarrow}(\mathbf{r})
\end{array}\right)\right]\right\}, \quad \alpha=x, y, z,
\end{aligned}
$$

and the vector potential by

$$
\begin{gathered}
\mathbf{A}_{x}=\sin (\theta+\phi) \nabla \omega-\cos (\theta+\phi) \sin \omega \cos \omega \nabla(\theta-\phi), \\
\mathbf{A}_{y}=\sin (\theta+\phi) \nabla \omega+\cos (\theta+\phi) \sin \omega \cos \omega \nabla(\theta-\phi), \\
\mathbf{A}_{z}=-\cos ^{2} \omega \nabla \theta-\sin ^{2} \omega \nabla \phi .
\end{gathered}
$$

$\hat{V}_{\mathbf{A}}$ contains the square of $\mathbf{A}$ and has the form of a potential energy operator:

$$
\hat{V}_{\mathrm{A}}=\int d \mathbf{r} \hat{\rho}(\mathbf{r}) V_{\mathrm{A}}(\mathbf{r})
$$

where

$$
V_{\mathbf{A}}(\mathbf{r})=\frac{\hbar^{2}}{2 m}\left[A_{x}^{2}(\mathbf{r})+A_{y}^{2}(\mathbf{r})+A_{z}^{2}(\mathbf{r})\right] .
$$

In order to analyze the noncollinear exchange and correlation functional

$$
E_{x c}[\rho, \mathbf{m}] \doteq F[\rho, \mathbf{m}]-T_{s}[\rho, \mathbf{m}]-\frac{1}{2} \int \frac{\rho(\mathbf{r}) \rho\left(\mathbf{r}^{\prime}\right)}{\left|\mathbf{r}-\mathbf{r}^{\prime}\right|}
$$

we study separately the noninteracting kinetic-energy functional $T_{s}[\rho, \mathbf{m}]$ and the universal energy functional $F[\rho, \mathbf{m}]$.

The noninteracting kinetic-energy functional

$$
T_{s}[\rho, \mathbf{m}] \doteq \min _{\Phi \rightarrow \rho, \mathbf{m}}\langle\Phi|\hat{T}| \Phi\rangle=\left\langle\Phi_{\rho, \mathbf{m}}|\hat{T}| \Phi_{\rho, \mathbf{m}}\right\rangle,
$$

where $\Phi_{\rho, \mathbf{m}}$ is the impure-spin KS state, is written in rotated spin space as

$$
T_{s}[\rho, \mathbf{m}]=\left\langle\Phi_{\rho, \mathbf{m}}\left|\hat{T}_{0}+\hat{T}_{\mathrm{A}}+\hat{V}_{\mathrm{A}}\right| \Phi_{\rho, \mathbf{m}}\right\rangle .
$$

We note that $M_{z}(\mathbf{r})$ represents in rotated spin-space the same magnetization density as $\mathbf{m}(\mathbf{r})$ in the laboratory (i.e., unrotated) spin space.

We further define

$$
T_{s}^{0}\left[\rho, M_{z}\right] \doteq \min _{\Phi \rightarrow \rho, M_{z}}\left\langle\Phi\left|\hat{T}_{0}\right| \Phi\right\rangle=\left\langle\Phi_{\rho, M_{z}}\left|\hat{T}_{0}\right| \Phi_{\rho, M_{z}}\right\rangle .
$$

$T_{s}^{0}\left[\rho, M_{z}\right]$ would be the collinear noninteracting kineticenergy functional if $M_{z}$ were a collinear magnetization density in the laboratory spin-space. The minimizing (KS) state $\Phi_{\rho, M_{z}}$ is pure spin. For weak A, we can approximate $T_{s}[\rho, \mathbf{m}]$ by $T_{s}^{0}\left[\rho, M_{z}\right]$ plus a correction: 


$$
\begin{aligned}
T_{s}[\rho, \mathbf{m}]= & T_{s}^{0}\left[\rho, M_{z}\right]+\left\langle\Phi_{\rho, M_{z}}\left|\hat{T}_{\mathrm{A}_{z}}\right| \Phi_{\rho, M_{z}}\right\rangle \\
& +\int d \mathbf{r} \rho(\mathbf{r}) V_{\mathrm{A}}(\mathbf{r})+\Delta T_{s}\left[\rho, M_{z}\right] .
\end{aligned}
$$

The first-order terms $\left\langle\Phi_{\rho, M_{z}}\left|\hat{T}_{\mathrm{A}_{x, y}}\right| \Phi_{\rho, M_{z}}\right\rangle$ vanish because $\Phi_{\rho, M_{z}}$ is pure spin and the operators $\hat{T}_{\mathrm{A}_{x, y}}$ flip a spin [Eqs. (57) and (58)]. The last term $\Delta T_{s}\left[\rho, M_{z}\right]$ contains second order and higher corrections in $\mathrm{A}$.

A similar analysis can be carried out for the internal energy functional,

$F[\rho, \mathbf{m}] \doteq \min _{\Psi \rightarrow \rho, \mathbf{m}}\left\langle\Psi\left|\hat{T}+\hat{V}^{\mathrm{ee}}\right| \Psi\right\rangle=\left\langle\Psi_{\rho, \mathbf{m}}\left|\hat{T}+\hat{V}^{\mathrm{e}}\right| \Psi_{\rho, \mathbf{m}}\right\rangle$.

$\Psi_{\rho, \mathbf{m}}$ is the minimizing state (impure spin). We then define

$$
F^{0}\left[\rho, M_{z}\right] \doteq \min _{\Psi \rightarrow \rho, M_{z}}\left\langle\Psi\left|\hat{T}_{0}+\hat{V}^{\mathrm{e}}\right| \Psi\right\rangle=\left\langle\Psi_{\rho, M_{z}}\left|\hat{T}_{0}+\hat{V}^{\mathrm{ee}}\right| \Psi_{\rho, M_{z}}\right\rangle .
$$

$F^{0}\left[\rho, M_{z}\right]$ would also be the collinear universal internal energy functional if $M_{z}$ were a collinear magnetization density in the laboratory spin space. The minimizing state $\Psi_{\rho, M_{z}}$ is pure spin. For weak $A$, we have

$$
\begin{aligned}
F[\rho, \mathbf{m}]= & F^{0}\left[\rho, M_{z}\right]+\left\langle\Psi_{\rho, M_{z}}\left|\hat{T}_{\mathrm{A}_{z}}\right| \Psi_{\rho, M_{z}}\right\rangle \\
& +\int d \mathbf{r} \rho(\mathbf{r}) V_{\mathrm{A}}(\mathbf{r})+\Delta F\left[\rho, M_{z}\right],
\end{aligned}
$$

where $\Delta F\left[\rho, M_{z}\right]$ contains second- and higher-order corrections in $\mathrm{A}$.

The noncollinear exchange and correlation functional can be expanded as follows:

$$
E_{x c}[\rho, \mathbf{m}]=E_{x c}^{0}\left[\rho, M_{z}\right]+E_{c}^{(1)}\left[\rho, M_{z}\right]+\Delta E_{x c}\left[\rho, M_{z}\right] .
$$

$E_{x c}^{0}\left[\rho, M_{z}\right]$ is the collinear exchange and correlation energy functional, where $M_{z}$ represents a collinear magnetization density in the laboratory spin space,

$E_{x c}^{0}\left[\rho, M_{z}\right]=F^{0}\left[\rho, M_{z}\right]-T_{s}^{0}\left[\rho, M_{z}\right]-\frac{1}{2} \int \frac{\rho(\mathbf{r}) \rho\left(\mathbf{r}^{\prime}\right)}{\left|\mathbf{r}-\mathbf{r}^{\prime}\right|}$,

$E_{c}^{(1)}\left[\rho, M_{z}\right]$ is a first-order correlation energy correction,

$E_{c}^{(1)}\left[\rho, M_{z}\right] \doteq\left\langle\Psi_{\rho, M_{z}}\left|T_{\mathrm{A}_{z}}\right| \Psi_{\rho, M_{z}}\right\rangle-\left\langle\Phi_{\rho, M_{z}}\left|T_{\mathrm{A}_{z}}\right| \Phi_{\rho, M_{z}}\right\rangle$,

and $\Delta E_{x c}\left[\rho, M_{z}\right]$ contains second- and higher-order corrections,

$$
\Delta E_{x c}\left[\rho, M_{z}\right]=\Delta F\left[\rho, M_{z}\right]-\Delta T_{s}\left[\rho, M_{z}\right] .
$$

In the noncollinear LSDA/GGA method used so far, ${ }^{8,9}$ instead of the noncollinear $E_{x c}[\rho, \mathbf{m}]$, one employs $E_{x c}^{0}\left[\rho, M_{z}\right]$. From Eqs. (69) and (71), the main first-order correction $E_{c}^{(1)}$ is given in terms of the pure-spin states $\Phi_{\rho, M_{z}}, \Psi_{\rho, M_{z}}$. Consequently, it is possible to obtain an LSDA approximation $E_{c L S D A}^{(1)}\left[\rho, M_{z}\right]$, using the spin-polarized uniform electron-gas model, in order to obtain a noncollinear LSDA exchange and correlation energy functional that takes into account the effect of $A$ to lowest order.
Recently, the formalism was developed to treat exchange exactly for noncollinear magnets. ${ }^{24}$ Preliminary applications show that noncollinearity of the spin density in exact exchange is enhanced compared with the result of noncollinear LSDA. ${ }^{8}$ This difference may be the result of the absence of correlation in the exact-exchange calculations, but it is equally possible that it is attributed to the missing term in the noncollinear LSDA exchange and correlation functional. It is intriguing to derive and observe the effect of the correction we have suggested and to compare results with the exactexchange scheme.

\section{SUMMARY}

Our study has addressed a mathematical issue in SDFT which accompanied the theory since it was first introduced. The question has been whether more than one spin potential may share the same ground state. If this were possible, the meaningfulness of the KS system would be in doubt. The latter is the noninteracting system, which is bound by an effective spin potential that forces its g.s. single-particle spin density to be the same as the interacting system's. If the noninteracting spin potential could not be determined uniquely, the KS system itself would not be unique.

For the collinear case, it has been shown already ${ }^{12,14}$ that apart from an arbitrariness of a spin constant in the spin potential and for systems which are not fully spin polarized, the ground state determines the diagonal spin potential uniquely.

The same question for noncollinear magnetic fields seemed harder but it is not. For systems with more than one electron, it actually reduces to whether a spin Hamiltonian can be locally rotated in spin space to spin-diagonal form. The main result of this paper is to show that this is only possible for collinear magnetic fields. Consequently, with the exception perhaps of pathological cases,${ }^{19}$ the invertibility of the mapping between spin potentials and g.s. spin densities is ensured both for collinear and for noncollinear magnetic fields.

As is often the case, our search to improve the mathematical foundations of the theory leads to better understanding and refining of the approximations used. Our study helps us identify a formal correction in the noncollinear exchange and correlation energy functional in LSDA/GGA to account for the vector potential $A$, which arises as the result of the rotation in spin space to diagonalize the spin density. The success of the noncollinear LSDA/GGA approximation as used so far indicates that the correction, for many systems, is probably small; however, this prediction must be tested.

\section{ACKNOWLEDGMENTS}

I am grateful to K. Capelle, H. Eschrig, W. Kohn, and W. E. Pickett for useful comments. I thank M. Levy for discussions and encouragement at an early stage. I thank J. Kübler for constructive comments on a previous version of this paper, which helped clarify the noncollinear LSDA/GGA approximation. 
*Electronic address: n.gidopoulos@rl.ac.uk

${ }^{1}$ P. Hohenberg and W. Kohn, Phys. Rev. 136, B864 (1964).

${ }^{2}$ W. Kohn and L. J. Sham, Phys. Rev. 140, A1133 (1965).

${ }^{3}$ M. Levy, Proc. Natl. Acad. Sci. U.S.A. 76, 6062 (1979).

${ }^{4}$ J. Kohanoff and N. I. Gidopoulos, Handbook of Molecular Physics and Quantum Chemistry (Wiley, Chichester, 2000), Vol. 2, Pt. 5, Chap. 26, p. 532.

${ }^{5}$ U. von Barth and L. Hedin, J. Phys. C 5, 1629 (1972).

${ }^{6}$ A. K. Rajagopal and J. Callaway, Phys. Rev. B 7, 1912 (1973).

${ }^{7}$ J. P. Perdew and A. Zunger, Phys. Rev. B 23, 5048 (1981).

${ }^{8} \mathrm{~J}$. Kübler, Theory of Itinerant Electron Magnetism (Clarendon, Oxford, 2000).

${ }^{9}$ L. M. Sandratskii, Adv. Mater. (Weinheim, Ger.) 47, 91 (1998).

${ }^{10}$ A. K. Rajagopal, Adv. Chem. Phys. 41, 59 (1980).

${ }^{11}$ K. Capelle and G. Vignale, Phys. Rev. Lett. 86, 5546 (2001).

${ }^{12}$ H. Eschrig and W. E. Pickett, Solid State Commun. 118, 123 (2001).

${ }^{13}$ N. Argaman and G. Makov, Phys. Rev. B 66, 052413 (2002).

${ }^{14}$ N. I. Gidopoulos, in The Fundamentals of Electron Density, Density Matrix and Density Functional Theory in Atoms, Molecules and the Solid State, edited by N. I. Gidopoulos and S. Wilson
(Kluwer, Dordrecht, 2003), p. 195.

${ }^{15}$ W. Kohn, A. Savin, and C. A. Ullrich, Int. J. Quantum Chem. 100, 20 (2004).

${ }^{16}$ C. A. Ullrich, Phys. Rev. B 72, 073102 (2005).

${ }^{17}$ For a three-dimensional vector $\mathbf{f}$, by $\mathbf{f} \cdot \boldsymbol{\sigma}$, we denote the $2 \times 2$ matrix $f_{x} \sigma_{x}+f_{y} \sigma_{y}+f_{z} \sigma_{z}$.

${ }^{18}$ N. I. Gidopoulos, Phys. Rev. B 57, 2146 (1998).

${ }^{19}$ Barring pathological cases, where the ground state of the spindiagonal part of $\mathcal{H}$ in Eq. (1) is also an eigenstate of its nondiagonal part, even though the two operators do not commute. See footnote 18 in Ref. 13.

${ }^{20}$ V. Korenman, J. L. Murray, and R. E. Prange, Phys. Rev. B 16, 4032 (1977).

${ }^{21}$ L. Kleinman, Phys. Rev. B 59, 3314 (1999).

${ }^{22}$ D. M. Bylander and L. Kleinman, Phys. Rev. B 60, R9916 (1999).

${ }^{23}$ K. Knöpfle, L. M. Sandratskii, and J. Kübler, Phys. Rev. B 62, 5564 (2000).

${ }^{24}$ S. Sharma, J. K. Dewhurst, C. Ambrosch-Draxl, S. Kurth, N. Helbig, S. Pitlalis, E. K. U. Gross, S. Shallcross, and L. Nordström, cond-mat/0510800 (unpublished). 